Supporting Information for Publication

\title{
Differential Isotope Labelling of 38 Dietary Polyphenols and their Quantification in Urine by Liquid Chromatography Electrospray lonization Tandem Mass Spectrometry
}

David Achaintre, ${ }^{\dagger}$ Audrey Buleté, ${ }^{\ddagger}$ Cécile Cren-Olivé, ${ }^{\ddagger}$ Liang $\mathrm{Li}, \$$ Sabina Rinaldi, ${ }^{\dagger}$ Augustin Scalbert ${ }^{*+}$

'Biomarkers Group, Nutrition and Metabolism Section, International Agency for Research on Cancer (IARC), 69372 Lyon Cedex 08, France

'Université de Lyon, Institut des Sciences Analytiques, UMR 5280 CNRS (Equipe TRACES), Université Lyon 1, ENS-Lyon, 5 rue de la Doua, 69100 Villeurbanne, France sDepartment of Chemistry, University of Alberta, Edmonton, Alberta T6G 2G2 Canada

\section{Content}

Table S1. Intra- and Inter-Batch Precision, Spike and Dilution Recovery for the Measurement of 38 Polyphenols. 
P a g e S2

Table S1. Intra- and Inter-Batch Precision, Spike and Dilution Recovery for the Measurement of 38

Polyphenols

\begin{tabular}{|c|c|c|c|c|c|c|c|}
\hline \multirow[t]{2}{*}{ Polyphenol } & \multirow[t]{2}{*}{ concentration $(\mu \mathrm{M})^{\mathrm{a}}$} & \multicolumn{2}{|c|}{ precision } & \multicolumn{4}{|c|}{ accuracy } \\
\hline & & $\begin{array}{c}\text { intra- } \\
\text { batch CV } \\
(\%)^{\mathrm{b}}\end{array}$ & $\begin{array}{c}\text { inter- } \\
\text { batch CV } \\
(\%)^{\mathrm{c}}\end{array}$ & $\begin{array}{c}\text { spike } \\
\text { recovery } \\
(\%)^{d}\end{array}$ & SD & $\begin{array}{l}\text { dilution } \\
\text { recovery } \\
(\%)^{\mathrm{e}}\end{array}$ & SD \\
\hline 4-hydroxybenzoic acid & $10.6-19.8$ & 5.0 & 8.4 & 106.9 & 10.3 & 97.8 & 6.5 \\
\hline 3-hydroxybenzoic acid & $0.113-0.505$ & 7.2 & 7.6 & 91.1 & 5.6 & 149.4 & 25.7 \\
\hline protocatechuic acid & $0.502-3.40$ & 5.4 & 6.4 & 96.7 & 3.9 & 116.5 & 10.9 \\
\hline vanillic acid & $4.52-19.7$ & 4.2 & 7.9 & 96.4 & 9.9 & 99.6 & 4.7 \\
\hline 3,5-dihydroxybenzoic acid & $2.85-7.97$ & 4.5 & 5.7 & 72.0 & 4.7 & 104.7 & 6.9 \\
\hline gallic acid & $<$ LOQ - 1.65 & 9.6 & 15.1 & 49.3 & 8.1 & 94.2 & 8.0 \\
\hline gallic acid ethyl ester & $<$ LOQ -4.87 & 9.1 & 7.3 & 96.7 & 6.0 & 107.2 & 11.6 \\
\hline 4-hydroxyphenylacetic acid & $54.5-869$ & 4.3 & 7.3 & 97.5 & 14.1 & 104.8 & 5.1 \\
\hline 3-hydroxyphenylacetic acid & $14.0-194$ & 4.7 & 6.3 & 83.3 & 15.3 & 101.4 & 5.6 \\
\hline 3,4-dihydroxyphenylacetic acid & $4.41-27.3$ & 4.3 & 7.5 & 73.2 & 5.4 & 107.4 & 7.5 \\
\hline homovanillic acid & $12.8-31.4$ & 4.0 & 5.5 & 84.1 & 11.0 & 116.9 & 11.3 \\
\hline 3,4-dihydroxyphenylpropionic acid & $0.437-13.5$ & 4.7 & 6.3 & 100.1 & 5.5 & 98.6 & 9.3 \\
\hline 3,5-dihydroxyphenylpropionic acid & $6.88-20.0$ & 6.8 & 8.4 & 103.2 & 8.8 & 84.2 & 4.9 \\
\hline$p$-coumaric acid & $0.101-3.25$ & 5.7 & 8.1 & 96.6 & 8.9 & 105.6 & 11.8 \\
\hline$m$-coumaric acid & $0.271-2.32$ & 4.1 & 10.6 & 99.1 & 2.6 & 100.2 & 10.9 \\
\hline caffeic acid & $0.463-5.08$ & 4.9 & 10.4 & 90.3 & 4.8 & 130.1 & 13.6 \\
\hline ferulic acid & $3.73-30.7$ & 3.9 & 7.0 & 87.5 & 14.9 & 101 & 6.9 \\
\hline kaempferol & $<$ LOQ -0.484 & 8.9 & 11.1 & 24.7 & 6.5 & 114.7 & 9.4 \\
\hline quercetin & $<\mathrm{LOQ}-0.528$ & 7.0 & 26.4 & 40.0 & 13.7 & 138.6 & 25.9 \\
\hline isorhamnetin & $0.466-0.537$ & 3.8 & 29.1 & 97.7 & 9.3 & $N D^{g}$ & N/A \\
\hline apigenin & $0.020-0.103$ & 7.5 & 25.1 & 90.8 & 7.8 & N/A & $\mathrm{N} / \mathrm{A}$ \\
\hline naringenin & $<$ LOQ - 8.03 & 6.5 & 9.8 & 88.5 & 3.5 & 124.6 & 14.5 \\
\hline hesperetin & $<$ LOQ - 8.06 & 9.3 & 8.2 & 84.3 & 4.7 & 112.3 & 7.5 \\
\hline daidzein & $<$ LOQ - 0.397 & 7.4 & 11.2 & 106.9 & 3.4 & 104 & 7.5 \\
\hline genistein & $0.052-0.195$ & 8.0 & 11.7 & 92.9 & 5.4 & 109.1 & 5.7 \\
\hline equol & $0.038-0.420$ & 6.1 & 7.5 & 99.8 & 3.4 & 107.2 & 9.8 \\
\hline phloretin & $<L O Q-3.79$ & 7.3 & 21.4 & 59.7 & 18.3 & 111.1 & 9.5 \\
\hline$(+)$-catechin & $<L O Q-0.963$ & 7.5 & 17.4 & 79.0 & 14.8 & N/A & $\mathrm{N} / \mathrm{A}$ \\
\hline (-)-epicatechin & $<\mathrm{LOQ}-8.26$ & 6.0 & 17.5 & 72.8 & 11.4 & 119.9 & 16.3 \\
\hline (+)-gallocatechin & $<L O Q$ & $N D^{g}$ & $N D^{g}$ & 63.5 & 17.4 & $N D^{g}$ & $\mathrm{~N} / \mathrm{A}$ \\
\hline (-)-epigallocatechin & $<$ LOQ -8.82 & 5.1 & 49.8 & 95.8 & 31.3 & 90.1 & 12.8 \\
\hline procyanidin dimers $B 1 / B 2^{f}$ & $<$ LOQ & $\mathrm{N} / \mathrm{A}$ & $\mathrm{N} / \mathrm{A}$ & 52.5 & 12.7 & $N D^{g}$ & $\mathrm{~N} / \mathrm{A}$ \\
\hline resveratrol & $<$ LOQ -0.772 & 7.5 & 10.0 & 77.5 & 8.4 & 114.3 & 12.0 \\
\hline tyrosol & $0.047-3.10$ & 4.1 & 7.5 & 94.3 & 1.7 & 102.9 & 4.2 \\
\hline hydroxytyrosol & $0.316-7.68$ & 4.9 & 7.9 & 98.4 & 7.7 & 112.5 & 11.0 \\
\hline enterodiol & $0.072-2.94$ & 5.0 & 6.0 & 99.6 & 8.1 & 94.1 & 3.9 \\
\hline enterolactone & $0.521-6.46$ & 3.9 & 5.2 & 100.3 & 4.1 & 103.1 & 7.6 \\
\hline
\end{tabular}


${ }^{a}$ Range of concentrations in urine samples used for validation.

${ }^{\mathrm{b}}$ Measured on 4 urine samples from the dietary intervention study.

${ }^{c}$ Measured on 2 samples from the dietary intervention study and the pooled urine sample from the EPIC study in 19 batches.

${ }^{d}$ Measured on 3 urine samples from the dietary intervention study (meal poor in polyphenols) spiked at 3 concentrations.

${ }^{e}$ Measured on 3 urine samples from the dietary intervention study at dilution $1 / 8,1 / 4$ and $1 / 2$.

fProcyanidin dimers B1 and B2 could not be distinguished in the chromatograms.

${ }^{9}$ Not determined because of too low concentrations. 